\title{
HD 46407: THE FIRST ECLIPSING-BINARY BARIUM STAR*
}

\author{
A. JORISSEN ${ }^{1}$, J. MANFROID ${ }^{2}$ and C. STERKEN ${ }^{3}$ \\ 1 European Southern Observatory, Garching bei München, Germany \\ 2 Institut d'Astrophysique, Université de Liège, Belgium \\ 3 Astrophysical Institute, University of Brussels (VUB), Belgium
}

\begin{abstract}
A long-term, high-accuracy, differential uvby monitoring of a sample of 19 barium stars revealed that HD 46407 , a well known barium star with an orbital period of $458 \mathrm{~d}$, is an eclipsing binary. The eclipse (of the companion by the barium star) was most clearly seen in November 1988, when a dip of 0.02 mag (in $y$ ) having a FWHM of about $10 \mathrm{~d}$ was recorded in the lightcurve. Confirmation of the eclipsing nature of HD 46407 was also obtained in February 1985 and in February 1990. A shallow secondary eclipse (extending over about $50 \%$ of the orbital period) seems to be present as well. The $b-y$ index is marginally variable, while $v-b$ color variations are correlated with the $y$ variations, in the sense that the system appears redder when it is fainter. We suggest that these variations originate in the scattering of the red-giant light by dust particles trapped (in a wide ring or shell?) around the (compact) companion. The eclipsed light would then be the light of the red giant backscattered by the dust around the companion.
\end{abstract}

\section{BARIUM STARS}

Barium stars are a family of peculiar red giant stars whose envelopes exhibit overabundances of carbon as well as of elements heavier than iron (see Smith, this conference, for a review). First identified by Bidelman \& Keenan (1951), they represent about $1 \%$ of all $\mathrm{G}$ and $\mathrm{K}$ red giants.

McClure (1983) found that a large fraction of barium stars belong to spectroscopic binaries. Jorissen \& Mayor (1988) showed that virtually all known southern barium stars with large barium overabundances are spectroscopic binaries, and McClure \& Woodsworth (1990) obtained orbital periods in the range of $80 \mathrm{~d}$ to more than $10 \mathrm{y}$. White dwarf (WD) companions are inferred from the mass function distribution, although very few of them can be detected directly from their UV radiation (Böhm-Vitense 1980, Dominy \& Lambert 1983, Böhm-Vitense, Nemec \& Proffitt 1984).

Mass transfer from the former primary towards the barium star, when the primary was a heavy element-rich star on the asymptotic giant branch (AGB), is believed to be responsible for the chemical anomalies displayed by barium stars (e.g. Boffin \& Jorissen 1988). The observation in many barium stars of a $10 \mu \mathrm{m}(N)$ excess, which intensity does not appear to be correlated with the other atmospheric peculiarities (Hakkila 1989), may point towards the presence in the system of dust left over from the mass transfer episode.

\section{PHOTOMETRY OF BARIUM STARS AT ESO}

The relatively large orbital separation (estimated as several AU) and the low luminosity of the companion (dictated by the SB1 nature of the system) imply that photometric variations related to the

"Based on observations carried out at the European Southern Observatory, La Silla, Chile 
binary nature of barium stars - if any - should be of rather small amplitude and have a long period. High accuracy and homogeneity of photometric data over periods of several years are therefore required for monitoring barium stars. Both requirements, together with good time coverage (4 to 6 months/year), are offered by the Long-Term Photometry of Variables (LTPV) program operating at ESO since 1982 (Sterken 1983).

The Strömgren uvby magnitudes of a sample of 19 barium stars have been monitored since July 1984 in a differential way: for each program star, two comparison stars were selected among nearby late-type giants. The frequency of observations is about two measurements per week during a typical one-month observing run. That frequency was increased to one measurement per night during three observing campaigns around the predicted times of eclipse of the companion of HD 46407 by the red giant (Sect. 3).

Observations were performed at the ESO $50 \mathrm{~cm}$ telescope (equipped with a RCA9789QB photomultiplier tube) or at the Danish $50 \mathrm{~cm}$ telescope (four-channel photometer with RCA9789QB tubes). The reduction to the standard uvby system is performed with the "multi-night" algorithm described by Manfroid (1985), taking into account the complete set of measurements obtained at the ESO 50 or Danish 50 telescopes since the beginning of the monitoring. In that way, offsets between observing runs are avoided, since there is only one color-transformation matrix for the whole set of measurements in a given system.

The accuracy of the differential magnitudes can be estimated from the standard deviations of the differential measurements of comparison pairs. For both the Danish 50 and ESO 50 systems (referred to in the following as D50 and E50-6, respectively, according to the notation of Jorissen, Manfroid \& Sterken 1991; see also Manfroid et al. 1991), $50 \%$ of these standard deviations are smaller than $0.003 \mathrm{mag}$ in the $y$ channel for observations spanning several years. The mean value of these standard deviations is somewhat smaller for the D50 system $(0.007 \mathrm{mag})$ than for the E50-6 system $(0.009 \mathrm{mag})$. More details about the observing procedure, the reduction method and the resulting accuracy can be found in Manfroid et al. (1991).

A detailed discussion of the results of the photometric monitoring for the sample of 19 barium stars is presented in Jorissen, Manfroid \& Sterken (1991). The main result of our monitoring is that the two barium stars with the shortest orbital periods in the sample, namely HD 121447 (185 d, Jorissen \& Mayor, in preparation) and HD 46407 (458.6 d; McClure \& Woodsworth 1990), are the only ones to present small, albeit significant, light variations. The observations of HD 46407, revealing the eclipsing-binary nature of that star, are presented in Sect. 3.

\section{HD 46407: THE FIRST ECLIPSING-BINARY BARIUM STAR}

In Fig. 1, all the available observations of HD 46407 (HR 2392, $y=6.27, b-y=0.66$, K0III Ba3) in the D50 system were phased according to McClure \& Woodsworth's (1990) spectroscopic ephemeris $(P=458.6 \pm 0.5 \mathrm{~d})$. The eclipse around phase 0.75 is clearly seen, although a more detailed analysis reveals that a better overlap between the measurements from the three eclipse campaigns can be achieved if one adopts a slightly different period of $452 \mathrm{~d}$. The origin of that discrepancy is not clear. A shallow secondary eclipse, corresponding to the transit of the companion in front of the barium star, may be present as well.

A clue to the origin of these light variations is provided by the behavior of the $v-b$ color index. Figure 2 shows that the system appears redder when it is fainter. The $b-y$ index exhibits the same behavior, although to a much lesser extent. We therefore suggest that the observed light variations originate in the scattering of the red-giant light by dust trapped around the companion. In particular, backscattering of the red-giant light by the dust makes the system look brighter and bluer during half of the orbital period. The eclipse itself would then correspond to the occultation of that backscattered light component when the dust passes behind the red giant. The eclipse is indeed about three times as long as would be expected for the eclipse of a point-like source by a red giant of radius $15 \mathrm{R}_{\odot}$ (in a $1.5 \mathrm{AU}$-wide orbit), indicating that the eclipsed light does not originate directly from the (WD?) companion but rather from some extended source. 


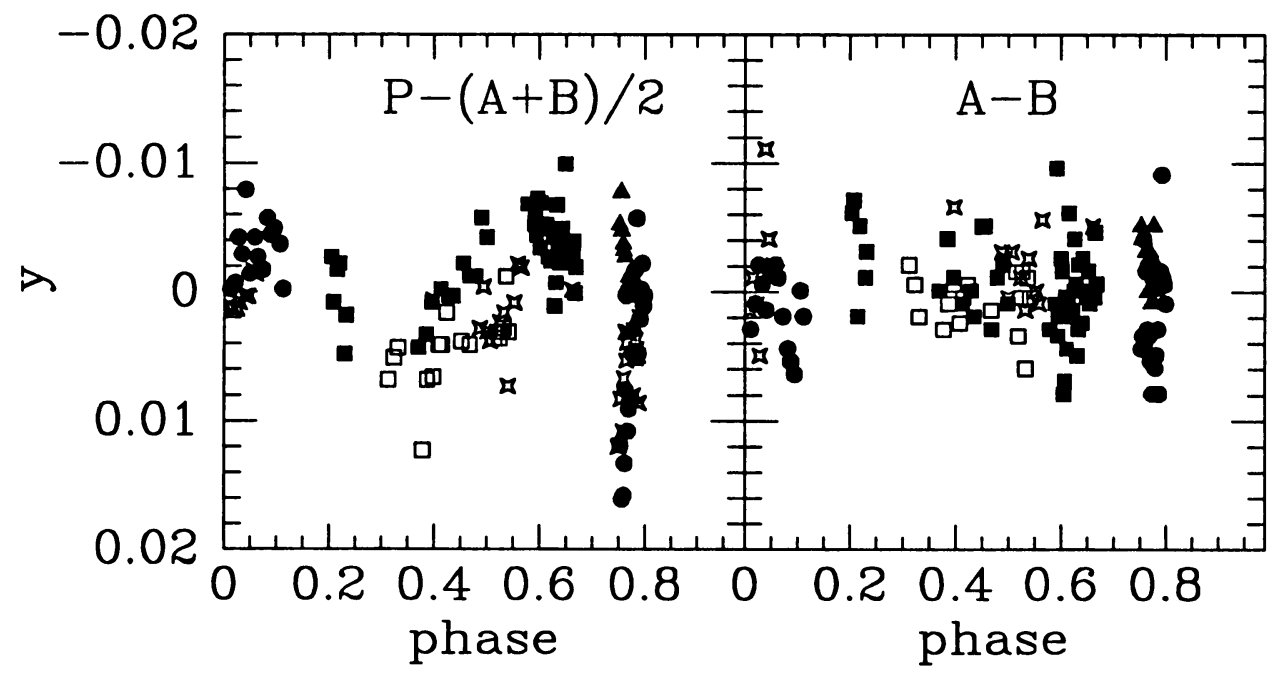

Figure 1: Phase diagram for the whole set of differential $y$ measurements in the D50 system (left panel: barium star relative to the comparison pair; right panel: comparison pair), adopting the spectroscopic elements from McClure \& Woodsworth (1990). The zero point of the magnitude scale corresponds to the mean value of the differential magnitude over the whole period considered. The

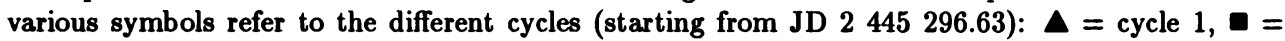
cycle $2, O=$ cycle $4, \star=$ cycle $5, \square=$ cycle 6 . Note the presence of a very wide secondary eclipse (transit of the companion in front of the barium star).

Although this model appears promising, it remains to be tested quantitatively. Also, the following questions need to be answered: (i) is the presence of dust inferred from the photometric behavior compatible with the lack of any conspicuous IR excess (Hakkila \& McNamara 1987, Hakkila 1989); (ii) how can dust survive (in a ring or shell?) for long periods of time (i.e. since the mass transfer episode) in the binary system (Poynting-Robertson effect; Poynting 1904, Robertson 1937)? (iii) what is the origin of the slight discrepancy between the photometric and spectroscopic periods?

Acknowledgments. We express our thanks to the observers of the LTPV program who carried out part of the observations. J.M. and C.S. acknowledge financial support from the Belgian Fund for Scientific Research (FNRS/NFWO). 


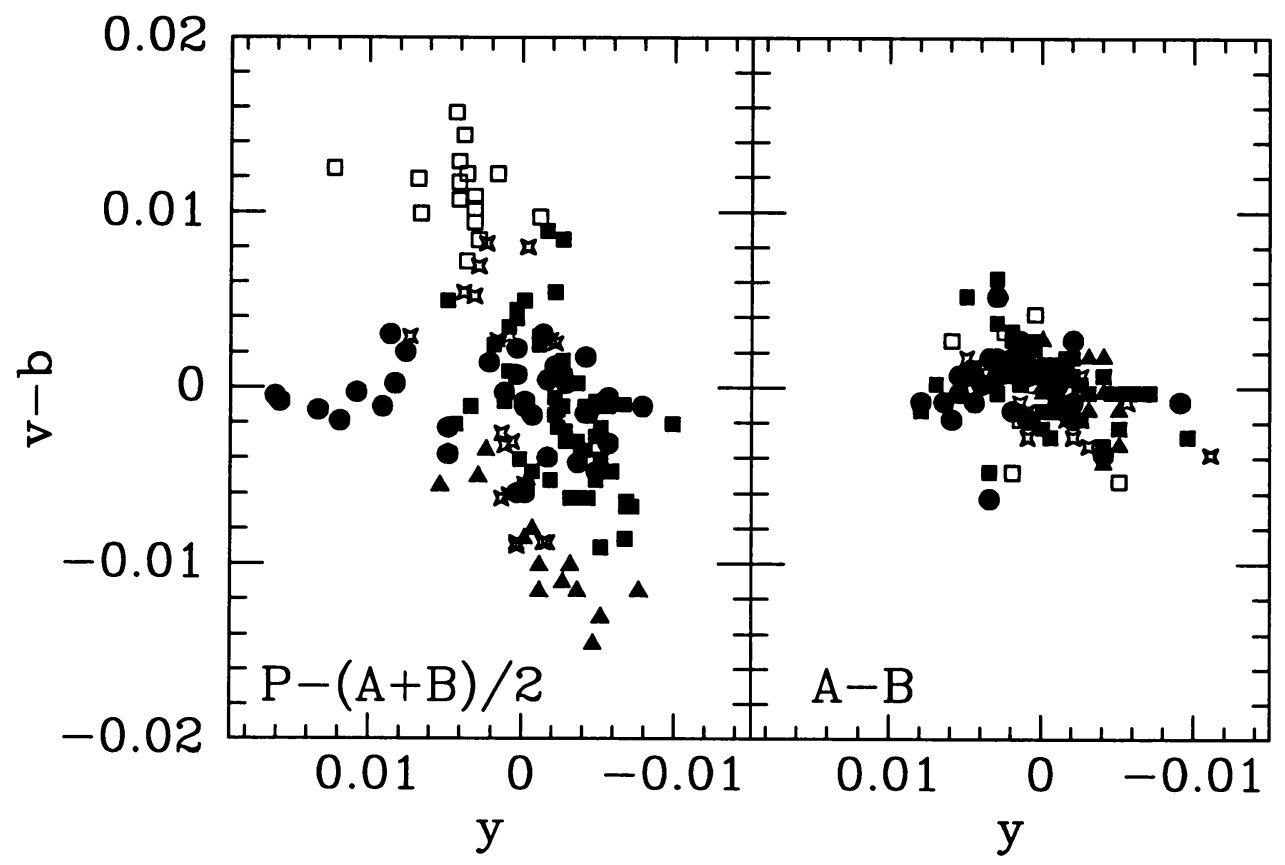

Figure 2: The $(y, v-b)$ diagram of the barium star (left panel) and the comparison pair (right panel). Symbols and magnitude zero points are as in Fig. 1. Note the triangles, which exhibit within a single observing run the general trend observed for the whole set of measurements. The filled circles standing to the left of that general trend correspond to the November 1988 eclipse.

\section{REFERENCES}

Bidelman, W.P., Keenan, P.C. 1951, ApJ, 114, 473

Boffin, H.M.J., Jorissen, A. 1988, A\&A, 205, 155

Böhm-Vitense, E. 1980, ApJ, 239, L79

Böhm-Vitense, E., Nemec, J., Proffitt, Ch. 1984, ApJ, 278, 726

Dominy, J.F., Lambert, D.L. 1983, ApJ 270, 180

Hakkila, J. 1989, A\&A, 213, 204

Hakkila, J., McNamara, B.J. 1987, A\&A, 186, 255

Jorissen, A., Mayor, M. 1988, A\&A, 198, 187

Jorissen, A., Manfroid, J., Sterken, C. 1991, A\&A, in press

Manfroid, J. 1985, habilit. thesis, Univ. of Liège

Manfroid, J., Sterken, C., Bruch, A., Burger, M., de Groot, M., Duerbeck, H., Duemmler, R., Figer, A., Hageman, T., Hensberge, H., Jorissen, A., Madejsky, R., Mandel, H., Ott, H., Reitermann, A., Schulte-Ladbeck, R., Stahl, O., Steenman, H., vander Linden D., Zickgraf, F.-J. 1991, A\&AS, 87, 481

McClure, R.D. 1983, ApJ, 268, 264

McClure, R.D., Woodsworth, A.W. 1990, ApJ, 352, 709

Poynting, J.H. 1904, Phil. Tran. Roy. Soc. London A 202, 525

Robertson, H.P. 1937, MNRAS 97, 423

Sterken, C. 1983, The Messenger, 33, 10 